Meeting report World Congress on Lung Health and 10th ERS Annual Congress
30 August-3 September 2000, Florence, Italy Pieter S Hiemstra

\author{
Department of Pulmonology, Leiden University Medical Center, Leiden, The Netherlands
}

Received: 9 October 2000

Accepted: 19 October 2000

Published: 6 November 2000
Respir Res 2000, 1:178-179

(c) Current Science Ltd (Print ISSN 1465-9921; Online ISSN 1465-993X)

\section{Pathogenesis of chronic obstructive pulmonary disease (COPD)}

The World Congress on Lung Health and 10th ERS Annual Congress brought together a wide range of scientists involved in studies on the pathogenesis of COPD. The research that was presented in various oral and poster presentations included a variety of approaches, including cell biology studies, analysis of lung tissue and studies of exhaled breath. The following report describes a selection of new developments related to research on the pathogenesis of COPD that were presented during the Florence meeting.

\section{Oxidative stress in COPD}

Whereas it is evident that cigarette smoke exposure results in oxidative stress, it is also clear that the inflammatory process observed in patients with COPD, asthma or cystic fibrosis, for example, results in disturbance of the oxidant-antioxidant balance. Several presentations focused on the alterations in cellular functioning that results from oxidative stress, the body's antioxidant systems and methods to detect oxidative stress in tissue and exhaled breath. From presentations of Barnes and coworkers (London, UK) and other research groups, it is clear that the analysis of exhaled breath condensate might form an attractive, non-invasive method for studying oxidative stress. However, it is also evident that many methodological issues still remain to be resolved before highly reproducible methods can be introduced. After the reports of some years ago on increased levels of hydrogen peroxide in exhaled breath condensate of patients with COPD, other markers of oxidative stress in breath condensate have now been introduced, including nitrosylated tyrosine residues. Data from the research group of Kharitonov and
Barnes (London, UK) in asthma patients revealed that nitrotyrosine in exhaled breath condensate is increased in patients with asthma, and decreased on treatment with high-dose steroids. Treatment of asthma patients or healthy controls with an inhaled nitric oxide (NO) synthesis inhibitor decreased both exhaled $\mathrm{NO}$ and nitrotyrosine in exhaled breath condensate, suggesting that nitrotyrosine in exhaled breath condensate results from endogenous (pulmonary) NO synthesis. In addition, levels of the lipid peroxidation marker 8-isoprostane too were increased in patients with inflammatory lung disease, and highest levels were found in patients with cystic fibrosis. It is interesting to note that orally administered antioxidants might not affect exhaled markers of oxidative stress.

\section{Metalloproteinases}

Various studies focused on the role of metalloproteinases and their inhibitors. Layton (Oxford, UK) gave a comprehensive review of the various members of the metalloproteinase family and their natural inhibitors, and discussed strategies for the development of inhibitors. A combined matrix metalloproteinase (MMP) and sheddase inhibitor was found to be active in rodent models of allergic asthma. D'Ortho (Créteil, France) discussed the role of MMPs in pulmonary vascular remodelling and pulmonary hypertension. Animal models for pulmonary hypertension were discussed in which MMP activity was altered by gene transfer of the MMP inhibitor tissue inhibitor of metalloproteinases-1 (TIMP-1) or TIMP-1 knockout mice. On the basis of these data the conclusion was drawn that MMPs might serve a protective role in pulmonary hypertension. These data seemed to be in contrast to recently published data in another animal model in which serine proteinase and MMP inhibitors

$\alpha_{1}-\mathrm{PI}=\alpha_{1}$-proteinase inhibitor; COPD $=$ chronic obstructive pulmonary disease; MMP $=$ matrix metalloproteinase; $\mathrm{NO}=$ nitric oxide; PDE $=$ phosphodiesterase; TIMP-1 = tissue inhibitor of metalloproteinases-1. 
were found to block vascular intimal thickening. Shapiro (St Louis, Missouri, USA) reviewed the well-known study from his laboratory showing the involvement of mouse macrophage elastase (MMP-12) in the development of cigarette-smoke-induced emphysematous lesions in mice. Subsequent studies now showed that treatment of mice in this model with a MMP inhibitor protects the mice against the development and progression of emphysema. Whereas these data suggest a predominant role for MMP in the development of smoke-induced emphysema, neutrophil elastase knockout mice too were found to develop less emphysema. These data suggest a role for both neutrophil-derived elastase and macrophage elastase in emphysema. In addition, the major role of neutrophil elastase in the antibacterial activity of the mouse neutrophil (that lacks the antimicrobial defensins that are characteristic of the antimicrobial armature of the neutrophil in other mammals and humans) was discussed.

\section{Inflammation in COPD}

There is evidence for the involvement of a wide range of cell types in the pathogenesis of COPD, including neutrophils, macrophages and $\mathrm{CD}^{+}{ }^{+} \mathrm{T}$ cells. Neutrophilic inflammation is observed in patients with $\alpha_{1}$-proteinase inhibitor $\left(\alpha_{1}-\mathrm{PI}\right)$ deficiency. Because $\alpha_{1}-\mathrm{PI}$ not only acts as an inhibitor of neutrophil serine proteinases such as neutrophil elastase, but also inhibits the cytotoxic and antimicrobial peptides neutrophil defensins, Wencker (Essen, Germany) studied neutrophil defensin levels in patients with $\alpha_{1}$-PI deficiency. Increased neutrophil defensin levels were found in the epithelial lining fluid of $\alpha_{1}$-PI deficiency patients, levels that correlated with neutrophil numbers and interleukin-8 levels. These observations lend further support to the hypothesis that neutrophil defensins might be one of the neutrophil products involved in the pathogenesis of emphysema associated with $\alpha_{1}$-PI deficiency. Therefore the treatment of patients with $\alpha_{1}-\mathrm{Pl}$ deficiency with $\alpha_{1}-\mathrm{Pl}$, for instance with the use of $\alpha_{1}-\mathrm{Pl}$ inhalation as reported by Vogelmeier (Munich, Germany), might potentially inhibit the proinflammatory activities of neutrophil defensins in addition to inhibiting neutrophil elastase.

The observations reported by Frankenberger (Munich, Germany) demonstrated an interesting approach to the study of macrophages in COPD. Analysis of induced sputum demonstrated that there is an increased proportion of $\mathrm{CD} 14^{+} \mathrm{CD} 16^{+}$macrophages in induced sputum from COPD patients. Because studies in peripheral blood have shown that $\mathrm{CD} 14^{+} \mathrm{CD} 16^{+}$monocytes represent a subpopulation of macrophages with a high proinflammatory activity, these studies indicate a novel approach to the study of macrophages in COPD. Eosinophilic inflammation has been found to be associated with exacerbations of chronic bronchitis. Qiu (London, UK) studied the expression of putative eosinophil chemotactic chemokines in exacerbations of chronic bronchitis, and showed an increase in eosinophil numbers and RANTES expression (shown by in situ hybridization) in bronchial biopsies of patients with chronic bronchitis during exacerbations. A trend towards an increase in MCP-4 and eotaxin expression was noted. The capacity of sputum from COPD patients to induce the migration of neutrophils in vitro across a double layer of cultured epithelial and endothelial cells was used by van Overveld (Antwerp, Belgium) to assess the anti-inflammatory effect of treatments with inhaled steroids or $\mathrm{N}$-acetylcysteine. Treatment of patients with these drugs reduced the extent of sputum-induced neutrophil transmigration. Whereas steroids were active after 2 months of treatment and were ineffective after prolonged treatment (more than 4 months), $\mathrm{N}$-acetylcysteine treatment was effective only after prolonged treatment (more than 4 months).

\section{Conclusion}

New treatment strategies for COPD are being developed and evaluated in preclinical and clinical models. Treatments such as those with antioxidants and with MMP or serine proteinase inhibitors were discussed elsewhere in this report. One other class of promising new drugs is the selective phosphodiesterase (PDE) inhibitors. These drugs are considered to be potentially useful in the treatment of COPD and asthma. Various studies described activities of selective PDE4 inhibitors that might further support their use in the treatment of COPD. A PDE4 inhibitor was found to inhibit mucin expression in an animal model of challenge with ovalbumin, whereas cell culture studies in vitro revealed inhibitory effects on processes such as smooth muscle proliferation in human airway and fibroblast-mediated collagen degradation. From these and similar studies, it is evident that appropriate cell culture and animal models might help in the development of novel and innovative ways to treat COPD.

Authors' affiliations: Department of Pulmonology, Leiden University Medical Center, Leiden, The Netherlands

Correspondence: Pieter S Hiemstra, PhD, Department of Pulmonology, Building 1 C3-P, Leiden University Medical Center, Albinusdreef 2, PO Box 9600, 2300 RC Leiden, The Netherlands. Tel: +31 71526 3848; fax: +3171526 6927; e-mail: P.S.Hiemstra@lumc.nl 